\title{
Loss of EGFR Mutation Expression Induced by NF1 Mutation in Non-Small Cell Lung Cancer: Non-Canonical Pathway of Tyrosine Kinase Inhibitors' Resistance
}

\author{
Jose Gabriel Negron Rodriguez*, Luis Orrego Poma, Manuel Leiva Galvez, Maria Claudia Rodriguez Zavaleta \\ and Wuilbert Rodriguez Pantigoso
}

Instituto Peruano De Oncologia Y Radioterapia [IPOR], Peru

Submission: February 06, 2021; Published: February 16, 2021

*Corresponding author: Jose Gabriel Negron Rodriguez, Instituto Peruano De Oncologia Y Radioterapia [IPOR], Av. Pablo Carriquiry 115 Urb. El Palomar- San Isidro - Lima, PE - Zipcode: 15036, Peru

\begin{abstract}
Non-small cell lung carcinoma (NSCLC) is considered the first cause of cancer-related death worldwide and many therapies have been developed against the presence of actionable mutations. For example, targeting the EGFR mutation has changed the overall prognosis in NSCLC. However, resistance to treatment has emerged and many canonical mechanisms have already been described. NF1 mutation causes partial or total loss of function of neurofibromin, which activates several intracellular pathways (MAPK / ERK, PI3K / AKT, TGF - $\beta$ / Smad), producing cellular proliferation, migration, apoptosis resistance and genetic instability, leading to loss of EGFR expression. Herein, we describe a case of a novel activation of a non-canonical pathway that led to treatment resistance by NF1 mutation.
\end{abstract}

Keywords: NSCLC; EGFR; NF1; PDL1

Abbreviations: NSCLC: Non-Small Cell Lung Cancer; NF1: Neurofibromin 1; TKI: Tyrosine Kinase Inhibitors; EMT: Epithelial-Mesenchymal Transition; EGFR: Epidermal Growth Factor Receptor; ALK: Anaplastic Lymphoma Kinase; TGF- $ß$ : Tumor growth factor type ß; MAPK: MitogenActivated Protein kinases; ERK: Extracellular Signal-Regulated kinases; PI3K: Phosphatidylinositol 3-kinases

\section{Introduction}

Lung cancer is the most prevalent cause of cancer related death worldwide [1,2]. Disease treatment will depend on three factors: first of all, clinical staging at diagnosis (I-III treatment with curative intent and IV treatment with palliative intent); secondly, neoplastic cellular type and finally, oncogenic molecular pathways of said specific cellular type [3]. In non-small cell lung cancer (NSCLC), the presence of mutations in epidermal growth factor receptor type 1 (EGFR) and the use of tyrosine kinase inhibitors (TKI) changed prognostic and survival in these patients [4]. With the above, it was established as favorable response factors: adenocarcinoma histology, women gender, non-smokers and Asian ethnicity [5-7]. However, the use of TKIs quickly led to the appearance of treatment resistance; there have been established some common or "canonical" resistance mechanisms such as: second mutations in EGFR such as substitution of methionine to threonine at location 790 (T790M); MET oncogene amplification, neuroendocrine transformation, PTEN loss, KRAS mutation, and epithelial-mesenchymal transition (EMT) $[8,9]$. Nevertheless, because of the complexity of the molecular and metabolic pathways in NSCLC, non-canonical resistant mechanisms can appear.

\section{Case Report}

We present the case of a 67-year-old woman of Asian descent, non-contributory pathological history, non-smoker. Clinically presents with demanding cough and wasting. Imaging studies reveal a heterogeneous hypodense solid lesion of $52 \mathrm{~mm}$ in greater diameter in the anterior segment of the right upper lobe, adjacent to the mediastinal pleura with loss of interface; associated with secondary nodules in both lung fields and secondary pretracheal, 


\section{Cancer Therapy \& Oncology International Journal}

retrocaval, precarinal, subcarinal and cervical adenopathies (group IV). Biopsy performed concluded: Non-small cell carcinoma: Adenocarcinoma positive for TTF1. EGFR and ALK studies were performed: EGFR mutation in Exon 21 (L858R) and negativity for ALK. Patient with diagnosis: NON-SMALL CELL LUNG CANCER (ADENOCARCINOMA) STAGE IVA (T3N3M1b) EGFRmut [EXON21 (L858R)] ALK (-). First-line treatment was started with second-generation TKI: Afatinib according to the NCCN guidelines and the LUX3 study $[3,10]$. At 3 months evaluation, she had partial response with decrease in the primary lesion; however, at 5 months; while being in response, she presented a new lymphadenopathy at cervical group $\mathrm{V}$ and pretracheoretrocaval region (Figure 1). The case was discussed at a tumor board with thoracic surgery and radiotherapy departments; concluded that the patient was not subject to radical oligometastatic treatment due to the proximity of lesions at the mediastinum. The patient continued with Afatinib, presented progressive bilateral lung disease after 10 months of treatment; at which time, a liquid biopsy was taken, obtaining as a result: Exon 19 deletion. Due to being oligosymptomatic, without evidence of T790M mutation and having a second generation TKI-sensitive driver mutation; patient continued therapy with Afatinib as stipulated in NCCN guidelines [3]. Thirteen months after starting treatment, the patient presented pericardial progression. A pericardial window was performed, obtaining a lower left lung lobe sample for pathological anatomy that concluded: Adenocarcinoma with positivity for TTF1, CK7 and p63. The patient clinically worsened, respiratory distress secondary to lymph node progression generating extrinsic compression of the pulmonary artery and the left main bronchus. Mediastinal radiotherapy is given: 30 Gy in VMAT technique in 10 fractions. A molecular platform (FoundationOne ${ }^{\circledR} \mathrm{CDx}$ ) of lung tissue was carried out, which concluded: mutation in the splicing site of NF1 (4431-1G>A), TP53 P152L mutation and PD-L1 100\% expression; moreover, loss of expression of EGFR mutation and absence of any oncogenic driver (ALK, BRAF, EGFR, ERBB2, KRAS, MET, RET, ROS1).

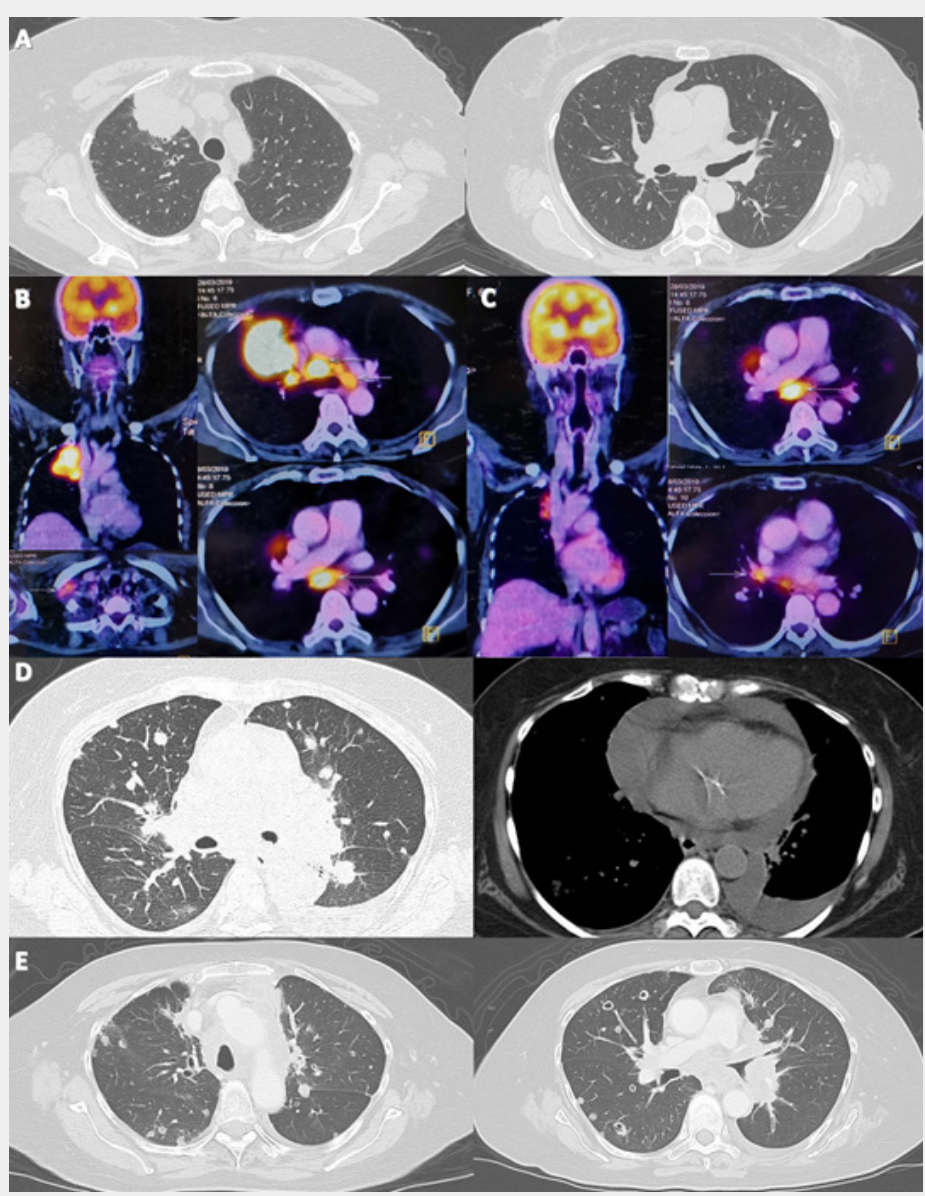

Figure 1:

A. CT at Onset of disease: upper right lobe $5 \mathrm{~cm}$ nodule, mediastinal lymphadenopathy

B. PETCT at Onset of disease: group IV cervical adenopathy

C. PETCT at 5 months: Group V cervical adenopathy and pretracheoretrocaval region

D. CT at 13 months: bilateral pulmonary nodules, pericardial effusion

E. CT at 14 months: extrinsic lymph node compression of the pulmonary artery and left main bronchus. 


\section{Cancer Therapy \& Oncology International Journal}

\section{Discussion}

Non-small cell lung carcinoma in its adenocarcinoma variant has become the target of various therapies due to its molecular complexity and the presence of actionable mutations [3,11]. The presence of EGFR mutations varies according to the population, representing an average of $10 \%$ of driver mutations in lung adenocarcinomas, and reaching up to $60 \%$ in the Asian population [11-13]. Thus, lung adenocarcinoma can be molecularly classified into positive oncogene and negative oncogene; positive oncogene adenocarcinomas present $11.3 \%$ mutations in EGFR and 32\% mutations in KRAS, as well as amplification of MET, HER2, ROS1, ALK and RET [11]. Negative oncogene adenocarcinomas show mutation of NF1, TP53 and RIT [11]. Molecular studies in EGFR mutated lung adenocarcinoma in Asian populations reveal high intratumoral heterogeneity, due to the presence of complete genome duplication events generally associated with mutations in TP53; although initially they are trunk mutations, later during their evolution, they allow the appearance of branched mutations that in the case of a mutated EGFR will lead to resistance to TKIs and expression of co-drivers, changing the natural history of the disease and appearance of resistance to treatment using pathways not previously described [13].

The canonical mechanisms of resistance to TKIs in patients with mutated EGFR are as follows: secondary mutations in EGFR such as substitution of methionine to threonine at location 790 (T790M); MET oncogene amplification, neuroendocrine transformation, PTEN loss, KRAS mutation, and epithelial-mesenchymal transition (EMT) $[8,9]$. The neurofibromatosis type 1 gene (NF1) is located on chromosome 17q11.2, consists of 60 exons and encodes for the protein: Neurofibromin, which acts as a tumor suppressor [14]; this, can be affected by various types of mutations (nonsense, missense, splicing site), any of which leads to coding a truncated protein and similar clinical scenarios [14,15]. NF1 has two functional domains: Sec14, which allows intracellular lipid and protein trafficking, and RasGAP (Ras-GRD), which, by increasing the hydrolysis rate of GTP, accelerates the conversion of the active form Ras-GTP to the inactive form Ras-GDP [14]. Moreover, NF1 has a role in the expression of tumor growth factor type $§$ (TGF- $ß$ ) and in immunoregulation $[14,16]$. Both TP53 and NF1 are found on chromosome 17 and have a role in tumorigenesis $[17,18]$ including oncogene negative lung adenocarcinoma $[11,19]$. NF1 mutations are loss-of-function mutations and serve as oncogenic drivers in this subtype of neoplasms $[11,19]$. Asian patients with NF1 mutations have disease-free survival and overall survival similar to patients with RAS mutations, this is explained by the activation of the mitogen-activated protein kinases (MAPK-ERK) pathway [19]. NF1 inactivation (initially demonstrated in colon cancer in studies with CRISPR/Cas9) leads to resistance to EGFR inhibition $[20,21]$. Subsequently, it was discovered that the null or low expression of NF1 in lung adenocarcinoma produced resistance to TKIs, activating the Ras and the MAPK pathway, with persistence of ERK phosphorylation despite EGFR blockade [22]. With this, the molecular basis for the use of a MEK inhibitor to decrease the activity and phosphorylation of ERK, added to the EGFR inhibitor, was postulated, obtaining a good response with this double inhibition [22].

Aberrant activation of the MEK / ERK pathway secondary to loss of NF1 is an important resistance mechanism, which can occur in the presence of primary or secondary EGFR mutations (including T790M) and becomes prevalent as an oncogenic driver in the presence of other disorders such as TP53 mutation [23]. The EGFR gene is located on chromosome 7p11.2 [24]. The two most common actionable mutations are the exon 19 deletion (delE746-A750) and a point mutation in codon 858 of exon 21, replacing leucine with arginine (L858R) [24]. Both correspond to 85-90\% of primary EGFR mutations [24]. In EGFR mutated lung adenocarcinoma, Her2 / Her3 signaling activation and constitutive or bypass activation of the PI3K / AKT pathway and the MAPK / ERK pathway lead to loss of EGFR expression [24,25], this is explained by the existence of genetic instability at the centromeric level in chromosome 7 (EGFR-ampch7 region), an effect mostly described in patients with Exon 19 deletion treated with TKIs [25]. Furthermore, in patients treated with second or third generation TKIs, they present loss of EGFR expression (independent of T790M mutation) due to molecular crosstalk between MAPK / ERK and PI3K / AKT pathways, as well as EMT phenotype [26]. The expression of PD-L1 can be increased by various mechanisms such as: activation of RAS, intrinsic activation of MAPK and PI3K, upregulation of inflammatory cytokines (TGF- $ß$ ) among others [27]; which leads to the activation of the TGF- $\beta$ / Smad pathway; the canonical EMT pathway $[27,28]$.

Our case presents a patient with Stage IV EGFR mutated (L858R) lung adenocarcinoma, treated with first line Afatinib; according to the LUX3 study [10], having good prognostic factors (Asian ethnicity, EGFR with common mutation, non-smoker) she should have had a median PFS of 11.4 months (13.6 months for being Asian), however the patient only had a 5 months PFS and clinical deterioration at 10 months after starting treatment (Figure 2). The liquid biopsy result, at the first progression, shows exon 19 deletion (and lack of T790M mutation), this phenomenon can be explained by a false positive or error in the test (specificity $96 \%$, sensitivity $98 \%$ for common mutations; specificity and sensitivity $70 \%$ for T790M) [29] or tumor heterogeneity [13]. The result of lung tissue sequencing at progression shows both NF1 and TP53 mutation (both correspond to negative NSCLC oncogene $[11,19]$ ), in addition to loss of expression of EGFR mutation, none oncogenic driver and high expression of PD-L1 (100\%). As described above, all NF1 mutations lead to truncated protein expression with partial or total loss of function [14], becoming an oncogenic driver [11,23]. RAS and MAPK / ERK pathways, are activated; that in the presence of blockade by a TKI will allow "crosstalk" and constitutive activation of the PI3K/AKT metabolic pathway 


\section{Cancer Therapy \& Oncology International Journal}

$[14,19,22,26]$. The double constitutive AKT-ERK activation will promote loss of EGFR expression due to genetic instability in the EGFR-ampch7 region of chromosome 7 [24-26]. Likewise, both the activation of RAS, MAPK / ERK and PI3K / AKT pathways and the increase in inflammatory cytokines such as TGF- $ß$ (generated by immunomodulation in mutated NF1) will cause upregulation of PD-L1 which will activate the EMT canonical pathway: TGF - $\beta$ / Smad $[14,27,28]$. It is through these molecular mechanisms that in the case of our patient a new non-canonical pathway of TKI resistance is expressed; linked to mutated NF1 that leads to the loss of expression of mutation in EGFR (Figure 3). This particular case, with a history of mutated EGFR expression that progresses to the use of TKI, and currently a high PD-L1 expressor, due to the mechanisms already described, would benefit from the use of the checkpoint inhibitor: Atezolizumab together with Carboplatin, Paclitaxel and Bevacizumab, as exposed in the IMpower150 study $[29,30]$, which to date is the only phase 3 study that includes this particular patient population.

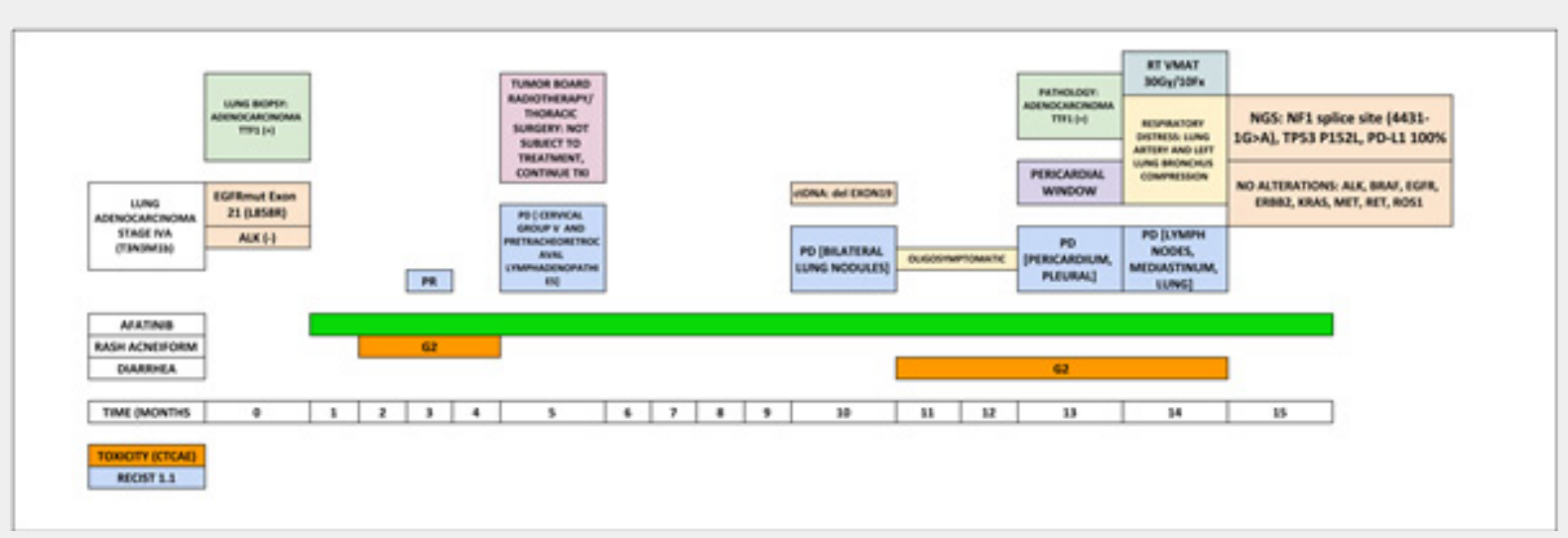

Figure 2: Timeline of disease.

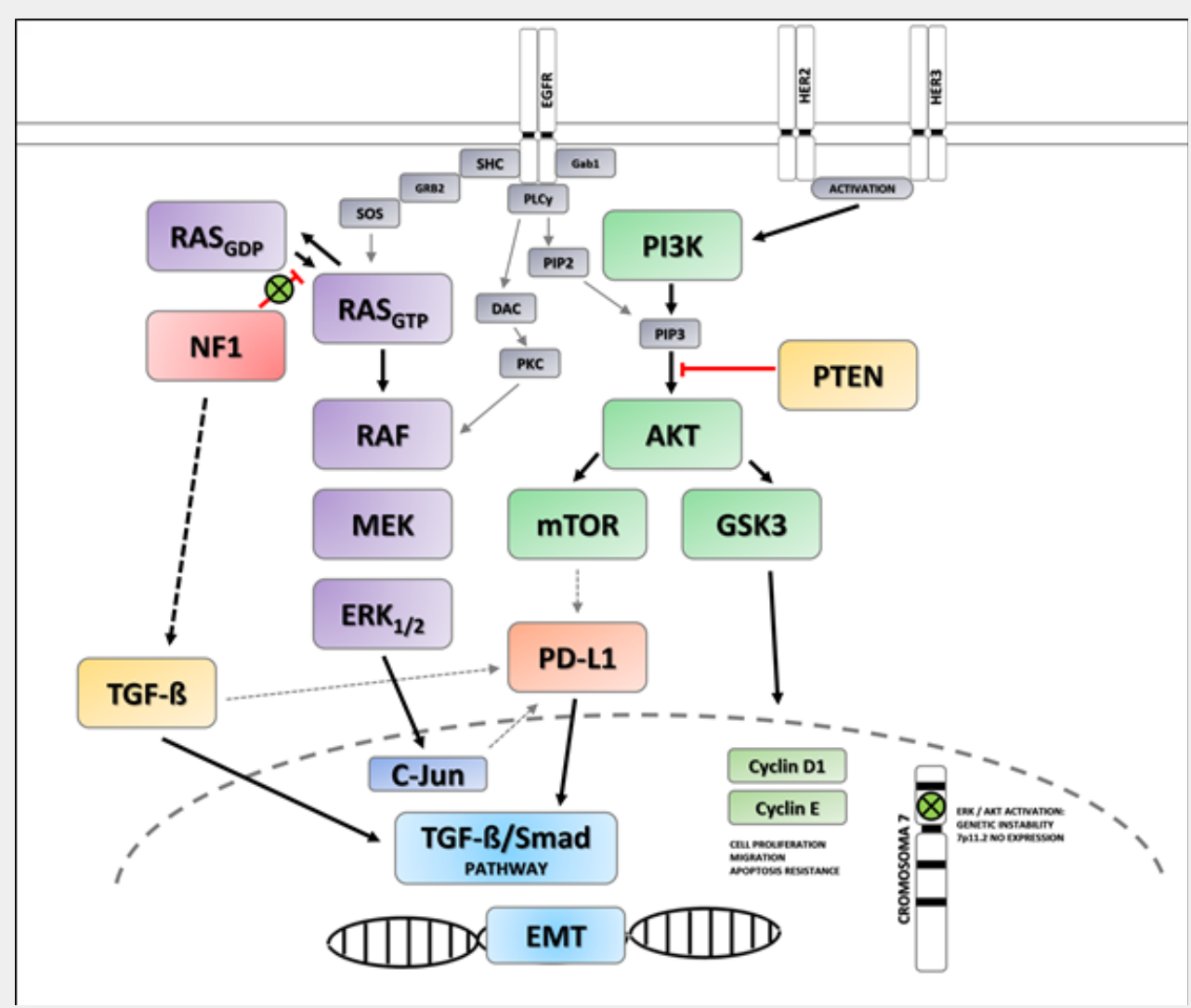

Figure 3: Constitutive activation of molecular pathways leads to loss of mutated EGFR expression. 


\section{Cancer Therapy \& Oncology International Journal}

\section{Conclusion}

NSCLC is a molecularly complex pathology, in which the presence of an actionable driver such as mutated EGFR defines a subgroup of patients with a supposedly favorable prognosis. We have observed and drafted the presence of a new non-canonical pathway of TKIs resistance. NF1 mutations contribute to the loss of expression of mutated EGFR (due to the activation of the RAS / MAPK / ERK and PI3K / AKT pathway), conditioning resistance to TKIs. Likewise, NF1 has a role in the expression of PD-L1 and activation of the canonical TGF- $\beta$ / Smad pathway of EMT by similar mechanisms. The NF1 mutation altered the disease natural history, became a poor prognostic factor, and produced novel resistance mechanisms linked to this alteration. Therefore, it is imperative to understand the metabolic and molecular pathways in the oncogenesis of the disease to provide individualized treatment according to the tumor molecular profile in patients with NSCLC.

\section{Acknowledgement}

Paula Alejandra Burela

\section{Conflict of Interest}

None.

\section{References}

1. Torre LA, Bray F, Siegel RL, Ferlay J, Lortet-Tieulent J, et al. (2015) Global cancer statistics, 2012: Global Cancer Statistics, 2012. CA Cancer J Clin 65(2): 87-108.

2. Siegel RL, Miller KD, Jemal A. Cancer statistics, 2020. CA Cancer J Clin 70(1): 7-30.

3. Ettinger D, Wood D, Aisner D, Akerley W, Bauman J (2020) National Comprehensive Cancer Network (NCCN). NCCN Clinical Practice Guidelines in Oncology. Non-Small Cell Lung Cancer (Version 8.2020).

4. Ono M, Kuwano M (2006) Molecular Mechanisms of Epidermal Growth Factor Receptor (EGFR) Activation and Response to Gefitinib and Other EGFR-Targeting Drugs. Clin Cancer Res 12(24): 7242-7251.

5. Gail MH, Eagan RT, Feld R, Ginsberg R, Goodell B, et al. (1984) Prognostic factors in patients with resected stage I non-small cell lung cancer. A report from the Lung Cancer Study Group. Cancer 54(9): 1802-1813.

6. Nakamura H, Ando K, Shinmyo T, Morita K, Mochizuki A, et al. (2011) Female Gender Is an Independent Prognostic Factor in Non-small-cell Lung Cancer: A Meta-analysis. Ann Thorac Cardiovasc Surg 17(5): 469-480.

7. D'Angelo SP, Janjigian YY, Ahye N, Riely GJ, Chaft JE, et al. (2012) Distinct Clinical Course of EGFR -Mutant Resected Lung Cancers: Results of Testing of 1118 Surgical Specimens and Effects of Adjuvant Gefitinib and Erlotinib. J Thorac Oncol 7(12): 1815-1822.

8. Huang L, Fu L (2015) Mechanisms of resistance to EGFR tyrosine kinase inhibitors. Acta Pharm Sin B 5(5): 390-401.

9. Morgillo F, Della Corte CM, Fasano M, Ciardiello F (2016) Mechanisms of resistance to EGFR-targeted drugs: lung cancer. ESMO Open 1(3): e000060.

10. Sequist LV, Yang JC-H, Yamamoto N, O’Byrne K, Hirsh V, et al. (2013)
Phase III Study of Afatinib or Cisplatin Plus Pemetrexed in Patients With Metastatic Lung Adenocarcinoma With EGFR Mutations. J Clin Oncol 31(27): 3327-3334.

11. The Cancer Genome Atlas Research Network (2014) Comprehensive molecular profiling of lung adenocarcinoma. Nature 511(7511): 543550 .

12. Zhao Z, Lin Y, Ng CSH, Zhang R, Wu X, et al. (2019) Mutation Profile of Resected EGFR -Mutated Lung Adenocarcinoma by Next-Generation Sequencing. The Oncologist 24(10): 1368-1374.

13. Nahar R, Zhai W, Zhang T, Takano A, Khng AJ, et al. (2018) Elucidating the genomic architecture of Asian EGFR-mutant lung adenocarcinoma through multi-region exome sequencing. Nat Commun 9(1): 216.

14. Le LQ Parada LF (2007) Tumor microenvironment and neurofibromatosis type I: connecting the GAPs. Oncogene 26(32): 4609-4616.

15. Castle B (2003) Evaluation of genotype-phenotype correlations in neurofibromatosis type 1. J Med Genet 40(10): 109e-109.

16. Yang F-C, Chen S, Clegg T, Li X, Morgan T, et al. (2006) Nf1+/- mast cells induce neurofibroma like phenotypes through secreted TGF- $\beta$ signaling. Hum Mol Genet 15(16): 2421-2437.

17. Cichowski K (1999) Mouse Models of Tumor Development in Neurofibromatosis Type 1\&nbsp; Science 286(5447): 2172-2176.

18. Vogel KS, Laura J Klesse, Susana Velasco-Miguel, Kimberly Meyers, Elizabeth J Rushing (1999) Mouse Tumor Model for Neurofibromatosis Type 1\&nbsp; Science 286(5447): 2176-2179.

19. Pan Y, Yuan C, Cheng C, Zhang Y, Ma Y, et al. (2019) Frequency and clinical significance of NF1 mutation in lung adenocarcinomas from East Asian patients: Frequency and clinical significance of NF1 mutation in lung adenocarcinomas from East Asian patients. Int J Cancer 144(2): 290-296.

20. Georgiou A, Stewart A, Cunningham D, Banerji U, Whittaker SR (2020) Inactivation of NF1 Promotes Resistance to EGFR Inhibition in KRAS/ NRAS/BRAF V600 -Wild-Type Colorectal Cancer. Mol Cancer Res 18(6): 835-846.

21. Post JB, Hami N, Mertens AEE, Elfrink S, Bos JL, et al. (2019) CRISPRinduced RASGAP deficiencies in colorectal cancer organoids reveal that only loss of NF1 promotes resistance to EGFR inhibition. Oncotarget 10(14): 1440-1457.

22. de Bruin EC, Cowell C, Warne PH, Jiang M, Saunders RE, et al. (2014) Reduced NF1 Expression Confers Resistance to EGFR Inhibition in Lung Cancer. Cancer Discov 4(5): 606-619.

23. Maertens O, Cichowski K (2014) Paths of Resistance to EGFR Inhibitors: Is NF Enough? Cancer Discov 4(5): 519-521.

24. Tabara K, Kanda R, Sonoda K, Kubo T, Murakami Y, et al. (2012) Loss of Activating EGFR Mutant Gene Contributes to Acquired Resistance to EGFR Tyrosine Kinase Inhibitors in Lung Cancer Cells. Shellman YG 7(7): e41017.

25. Furugaki K, Iwai T, Moriya Y, Harada N, Fujimoto-Ouchi K (2014) Loss of an EGFR-amplified chromosome 7 as a novel mechanism of acquired resistance to EGFR-TKIs in EGFR-mutated NSCLC cells. Lung Cancer 83(1): 44-50.

26. Xu J, Zhao X, He D, Wang J, Li W, et al. (2018) Loss of EGFR confers acquired resistance to AZD9291 in an EGFR-mutant non-small cell lung cancer cell line with an epithelial-mesenchymal transition phenotype. J Cancer Res Clin Oncol 144(8): 1413-1422.

27. Cha J-H, Chan L-C, Li C-W, Hsu JL, Hung M-C (2019) Mechanisms Controlling PD-L1 Expression in Cancer. Mol Cell 76(3): 359-370. 


\section{Cancer Therapy \& Oncology International Journal}

28. Zhang Y, Zeng Y, Liu T, Du W, Zhu J, et al. (2019) The canonical TGF- $\beta$ / Smad signalling pathway is involved in PD-L1-induced primary resistance to EGFR-TKIs in EGFR-mutant non-small-cell lung cancer. Respir Res 20(1): 164.

29. Oxnard GR, Thress KS, Alden RS, Lawrance R, Paweletz CP, et al. (2016) Association Between Plasma Genotyping and Outcomes of Treatment with Osimertinib (AZD9291) in Advanced Non-Small-Cell Lung Cancer J Clin Oncol 34(28): 3375-3382.

30. Socinski MA, Jotte RM, Cappuzzo F, Orlandi F, Stroyakovskiy D, et al. (2018) Atezolizumab for First-Line Treatment of Metastatic Nonsquamous NSCLC. N Engl J Med 378(24): 2288-2301.
Your next submission with Juniper Publishers will reach you the below assets

- Quality Editorial service

- Swift Peer Review

- Reprints availability

- E-prints Service

- Manuscript Podcast for convenient understanding

- Global attainment for your research

- Manuscript accessibility in different formats ( Pdf, E-pub, Full Text, Audio)

- Unceasing customer service

Track the below URL for one-step submission https://juniperpublishers.com/online-submission.php 